\title{
A note on subdirectly irreducible rings
}

\section{Madhukar G.. Deshpande}

It is proved that every subdirectly irreducible ring can be obtained as a proper homomorphic image of another subdirectly irreducible ring. An example of a subdirectly irreducible ring $R$ with heart $H$ is given for which

i) $R$ has a non-subdirectly irreducible homomorphic image,

ii) $R / H$ is an integral domain, and

iii) the commutator ideal $C(R)$ of $R$ coincides with $R$.

In a recent issue of this Bulletin [3], H.G. Moore has constructed an example of a subdirectly irreducible ring which has a non-subdirectly irreducible homomorphic image. Nevertheless, this ring has certain 'not too bad' properties such as the factor ring modulo the heart is boolean and the commutator ideal of the ring is nil. In the present note we first prove that any subdirectly irreducible ring can be obtained as a homomorphic image of another subdirectly irreducible ring and then point out an example of a subdirectly irreducible ring which is very different in nature from the one constructed by Moore.

1.

We restrict ourselves to rings with identity. This restriction is not too severe because it is known [2, page 648] that a subdirectly irreducible ring can be extended to one with identity.

PROPOSITION. Given a subdirectly irreducible ring $R$, there exists a subdirectly irreducible ring $S$ such that $R$ is a proper homomorphic image of $S$.

Received 22 August 1970. 
Proof. Let $H$ denote the heart of $R$. Let $S$ be the ring defined by $(X ;+)=R \oplus R$, while multiplication obeys the rule $(a, b)(c, d)=(a c, b c+a d)$. We claim that $S$ is subdirectly irreducible with heart $K=\{(0, h) \mid h \in H\}$. For, suppose $(a, b)$ is a nonzero element of $S$. If $a \neq 0$ then for some $x, y, u, v \in R, a x+y a+u a v$ is a nonzero element of $H$. Then

$$
(a, b)(0, x)+(0, y)(a, b)+(0, u)(a, b)(v, 0)=(0, a x+y a+u a v)
$$

is a nonzero element of $K$. If however $a=0$, then $b \neq 0$ and again, for some $p, q, x, s \in R$ we must have a nonzero element $b p+q b+r b s$ of $H$. Then, accordingly

$$
(0, b)(p, 0)+(q, 0)(0, b)+(r, 0)(0, b)(s, 0)=(0, b p+q b+r b s)
$$

is a nonzero element of $K$. Thus $S$ is subdirectly irreducible with heart $K$. Now, the mapping $\eta: S \rightarrow R$ defined by $(a, b) \rightarrow a$ is a homomorphism of $S$ onto $R$ with kernel $T=\{(0, b) \mid b \in R\}$. This proves the result.

REMARK. Note that $S$ contains an isomorphic copy of $R$ since $a \leftrightarrow(a, 0)$ is a monomorphism.

\section{2.}

The author [1] has defined a right subdirectly irreducible (RSI) ring as one in which the intersection of all nonzero right ideals $H$ is nonzero. It can be shown [1] that a RSI ring $R$ is in fact subdirectly irreducible and $H$ is the heart of $R$. We now construct an example of a RSI (and hence subdirectly irreducible) ring $R$ for which

i) a homomorphic image is not subdirectly irreducible,

ii) $R / H$ is an integral domain (which is far from a boolean ring) and for which,

iii) the commutator ideal $C(R)$ contains units (and hence equals $R$ ).

EXAMPLE. Let $D_{0}$ be an arbitrary division ring and $D_{0}\left[x_{1}\right]$ the polynomial ring in an indeterminate. It can be shown that $D_{0}\left[x_{1}\right]$ is a 
left and right Ore domain and $D_{1}=\left\{f g^{-1} \mid f, g \in D_{0}\left[x_{1}\right], g \neq 0\right\}$ is its quotient division ring. Define inductively $D_{n+1}$ as the quotient division ring of the Ore domain $D_{n}\left[x_{n+1}\right]$ and let $D=\bigcup_{i=1}^{\infty} D_{i} \cdot \quad D$ is then a division ring. Let $D[x]$ be the ring of polynomials in an indeterminate $x$. Define $\sigma: D[x] \rightarrow D$ by $\sigma\left[f\left(x, x_{1}, \ldots, x_{n}\right)\right]=f\left(x_{1}, x_{2}, \ldots, x_{n+1}\right)$ and $\eta: D[x] \rightarrow D$ by $\eta\left[f\left(x, x_{1}, \ldots, x_{n}\right)\right]=f\left(0, x_{1}, \ldots, x_{n}\right)$ where $f$ denotes a function of the variables indicated with coefficients in $D_{0}$. We note that $\sigma$ is a monomorphism and $\eta$ is an epimorphism. We now define $R$ to be the ring such that $(R,+)=D[x] \oplus D$ with multiplication in $R$ given by $(a, b)(c, d)=(a c, b \eta(c)+\sigma(a) d)$. If $H=\{(0, b) \mid b \in D\}$, then it can be seen that $R$ is subdirectly irreducible (in fact, RSI) with heart $H$ and that $R / H \cong D[x]$ which is an integral domain. If $a, b \in D_{0}$, such that $a b-b a=u \neq 0$, then in $R$, we have $(a, 0)(b, 0)-(b, 0)(a, 0)=(u, 0)$ which is a unit since $(u, 0)\left(u^{-1}, 0\right)=\left(u^{-1}, 0\right)(u, 0)=(1,0)$. This shows that the commutator ideal $C(R)$ of $R$ contains the identity. Also $D[x]$ is not subdirectly irreducible and hence $R$ has a homomorphic image $R / H$ which is not subdirectly irreducible.

\section{References}

[1] Madhukar G. Deshpande, "Structure of right subdirectly irreducible rings I", J. Algebra (to appear).

[2] Nathan Divinsky, "Commutative subdirectly irreducible rings", Proc. Amer. Math. Soc. 8 (1957), 642-648. 
[3] H.G. Moore, "Subdirectly irreducible rings - some pathology", Bull. Austral. Math. Soc. I (1969), 353-355.

Marquette University,

Milwaukee,

Wisconsin, USA. 